\title{
Molecular content of the circumstellar disk in AB Aurigae (Research Note)
}

\section{First detection of SO in a circumstellar disk}

\author{
A. Fuente ${ }^{1}$, J. Cernicharo ${ }^{2}$, M. Agúndez ${ }^{3}$, O. Berné ${ }^{4}$, J. R. Goicoechea ${ }^{2}$, T. Alonso-Albi ${ }^{1}$, and N. Marcelino ${ }^{2}$ \\ ${ }^{1}$ Observatorio Astronómico Nacional (OAN), Apdo. 112, 28803 Alcalá de Henares, Madrid, Spain \\ e-mail: a.fuente@oan.es \\ 2 Departamento de Astrofísica, Centro de Astrobiología (CSIC-INTA), Crta Ajalvir km 4, 28850 Madrid, Spain \\ ${ }^{3}$ LUTH, Observatoire de Paris-Meudon, 5 place Jules Janssen 92190 Meudon, France \\ ${ }^{4}$ Leiden Observatory, Leiden University, PO Box 9513, 2300 RA Leiden, The Netherlands
}

Received 30 April 2010 / Accepted 27 September 2010

ABSTRACT

\begin{abstract}
Aims. Very few molecular species have been detected in circumstellar disks surrounding young stellar objects. We are carrying out an observational study of the chemistry of circumstellar disks surrounding T Tauri and Herbig Ae stars. First results of this study are presented in this note.

Methods. We used the EMIR receivers recently installed at the IRAM $30 \mathrm{~m}$ telescope to carry out a sensitive search for molecular lines in the disks surrounding AB Aur, DM Tau, and LkCa 15.

Results. We detected lines of the molecules $\mathrm{HCO}^{+}, \mathrm{CN}, \mathrm{H}_{2} \mathrm{CO}, \mathrm{SO}, \mathrm{CS}$, and $\mathrm{HCN}$ toward $\mathrm{AB}$ Aur. In addition, we tentatively detected $\mathrm{DCO}^{+}$and $\mathrm{H}_{2} \mathrm{~S}$ lines. The line profiles suggest that the $\mathrm{CN}, \mathrm{HCN}, \mathrm{H}_{2} \mathrm{CO}, \mathrm{CS}$, and $\mathrm{SO}$ lines arise in the disk. This makes it the first detection of SO in a circumstellar disk. We have unsuccessfully searched for SO toward DM Tau and LkCa 15, and for c- $\mathrm{C}_{3} \mathrm{H}_{2}$ toward $\mathrm{AB}$ Aur, DM Tau, and LkCa 15. Our upper limits show that contrary to all molecular species observed so far, SO is not as abundant in DM Tau as it is in AB Aur.

Conclusions. Our results demonstrate that the disk associated with AB Aur is rich in molecular species. Our chemical model shows that the detection of $\mathrm{SO}$ is consistent with that expected from a very young disk where the molecular adsorption onto grains does not yet dominate the chemistry.
\end{abstract}

Key words. stars: formation - stars: individual: AB Aur - stars: pre-main sequence - stars: variables: T Tauri, Herbig Ae/Be circumstellar matter - protoplanetary disks

\section{Introduction}

Circumstellar disks are complex systems in which essentially all processes that play a role in the interstellar medium, such as UV radiation, X-rays, grain surface chemistry, molecular depletion, turbulent mixing, accretion flows, and time dependency, are at work. Chemical models with increasing complexity have been developed in the last decade (see e.g. Aikawa et al. 2000; Dutrey et al. 2007; Agúndez et al. 2008; Nomura et al. 2009), but the disk chemistry is a quite unexplored field from the observational point of view. Large millimeter telescopes have started to provide some insight into the chemistry of the cold gas toward the most massive nearby disks. Thus far, few molecules $\left(\mathrm{CO},{ }^{13} \mathrm{CO}, \mathrm{CN}, \mathrm{C}_{2} \mathrm{H}, \mathrm{HCN}, \mathrm{HNC}, \mathrm{HCO}^{+}, \mathrm{H}_{2} \mathrm{CO}\right)$ have been detected in circumstellar disks. This small molecular inventory is mainly owing to the weakness of the molecular emission from circumstellar disks. Disks have small masses, lower than $0.1 M_{\odot}$, small sizes, radii of a few $100 \mathrm{AU}$, and because of depletion in the midplane and/or photodissociation in the surface, the disk averaged abundances of most molecules (including $\mathrm{CO}$ and its isotopologues) are a factor of 5-10 lower than in the interstellar medium. High sensitivity is therefore required for an observational study. We have carried out a sensitive search for molecular lines mainly in the disk around the Herbig Ae star AB Aur using the IRAM $30 \mathrm{~m}$ telescope. Some lines have also been searched toward DM Tau and LkCa 15. Our results show the rich molecular content in the disk around AB Aur.

$\mathrm{AB}$ Auriga is one of the nearest, brightest, and best studied Herbig Ae stars. It has a spectral type A0-A1 (Hernández et al. 2004) and is located to the southwest of the molecular cloud L1517 (Duvert et al. 1986), at a distance of 145 pc (van den Ancker et al. 1998). Interferometric observations at millimeter wavelengths detected the circumstellar disk around this star in the continuum and in the $\mathrm{CO}$ (and its isotopologues) lines (Piétu et al. 2005). Instead of being centrally peaked, the continuum emission is dominated by a bright, asymmetric (spiral-like) feature at about $140 \mathrm{AU}$ from the central star.

The disk modeling of the continuum and molecular emission showed that the disk is warm and showed no evidence of CO depletion. Schreyer et al. (2008) searched for emission of the $\mathrm{HCO}^{+} 1 \rightarrow 0, \mathrm{HCN} 1 \rightarrow 0, \mathrm{CS} 2 \rightarrow 1, \mathrm{C}_{2} \mathrm{H} 1 \rightarrow 0$ and some $\mathrm{CH}_{3} \mathrm{OH}$ lines in this disk using the Plateau de Bure Interferometer (PdBI), but they only detected the $\mathrm{HCO}^{+} 1 \rightarrow$ 0 line.

\section{Observations}

The list of observed lines and the telescope parameters are given in Table 1. The observations were done in two observing periods, 
Table 1. List of targeted lines.

\begin{tabular}{lllll}
\hline \hline & \multicolumn{1}{c}{ Line } & Freq. (GHz) & $H P B W\left({ }^{\prime \prime}\right)$ & $\eta_{b}$ \\
\hline $\mathrm{CO}$ & $2 \rightarrow 1$ & 230.538 & 10 & 0.63 \\
$\mathrm{HCO}^{+}$ & $1 \rightarrow 0$ & 89.188 & 28 & 0.81 \\
$\mathrm{HCO}^{+1}$ & $3 \rightarrow 2$ & 267.558 & 9 & 0.53 \\
$\mathrm{HCN}$ & $1 \rightarrow 0$ & 88.631 & 28 & 0.81 \\
$\mathrm{HCN}$ & $3 \rightarrow 2$ & 265.886 & 9 & 0.53 \\
$\mathrm{CN}$ & $1 \rightarrow 0$ & 113.490 & 22 & 0.81 \\
$\mathrm{CN}$ & $2 \rightarrow 1$ & 226.874 & 10 & 0.63 \\
$\mathrm{CS}$ & $2 \rightarrow 1$ & 97.981 & 25 & 0.81 \\
$\mathrm{CS}$ & $3 \rightarrow 2$ & 146.969 & 16 & 0.74 \\
$\mathrm{C}_{2} \mathrm{H}$ & $3 \rightarrow 2$ & 262.004 & 9 & 0.53 \\
$\mathrm{H}_{2} \mathrm{CO}$ & $3_{0,3} \rightarrow 2_{0,2}$ & 218.222 & 11 & 0.63 \\
$\mathrm{SO}$ & $3_{4} \rightarrow 2_{3}$ & 138.178 & 17 & 0.74 \\
$\mathrm{SO}$ & $5_{6} \rightarrow 45$ & 219.949 & 11 & 0.63 \\
$\mathrm{c}-\mathrm{C}_{3} \mathrm{H}_{2}$ & $2_{1,2} \rightarrow 1_{0,1}$ & 85.339 & 29 & 0.81 \\
$\mathrm{c}-\mathrm{C}_{3} \mathrm{H}_{2}$ & $6_{0,6} \rightarrow 5_{1,5}$ & 217.822 & 11 & 0.63 \\
$\mathrm{c}-\mathrm{C}_{3} \mathrm{H}_{2}$ & $6_{1,6} \rightarrow 5_{0,5}$ & 217.822 & 11 & 0.63 \\
$\mathrm{DCO}$ & $2 \rightarrow 1$ & 144.077 & 16 & 0.74 \\
$\mathrm{DCN}$ & $2 \rightarrow 1$ & 144.828 & 16 & 0.74 \\
$\mathrm{SiO}$ & $2 \rightarrow 1$ & 86.847 & 29 & 0.81 \\
$\mathrm{SiO}$ & $6 \rightarrow 5$ & 260.518 & 9 & 0.53 \\
$\mathrm{HCO}$ & $1_{0,1} 3 / 2,2 \rightarrow 0_{0.0} 1 / 2,1$ & 86.671 & 29 & 0.81 \\
$\mathrm{HCO}$ & $1_{0,1} 3 / 2,1 \rightarrow 0_{0.0} 1 / 2,0$ & 86.708 & 29 & 0.81 \\
$\mathrm{HCO}$ & $1_{0,1} 1 / 2,1 \rightarrow 0_{0.0} 1 / 2,1$ & 86.777 & 29 & 0.81 \\
$\mathrm{HCO}$ & $1_{0,1} 1 / 2,0 \rightarrow 0_{0.0} 1 / 2,1$ & 86.805 & 29 & 0.81 \\
$\mathrm{H}_{2} \mathrm{~S}$ & $1_{1,0} \rightarrow 1_{0,1}$ & 168.763 & 14 & 0.74 \\
\hline & & & & \\
\hline & & &
\end{tabular}

Notes. (1) Observed with the wide band spectrometer WILMA in the HCN $3 \rightarrow 2$ tuning. (2) Observed with the wide band spectrometer WILMA in the $\mathrm{c}-\mathrm{C}_{3} \mathrm{H}_{2} 6_{0,6} \rightarrow 5_{1,5}$ tuning.

September 2009 and March 2010, with the new EMIR receivers arranged to provide a bandwidth of $4 \mathrm{GHz}$ in both, the $3 \mathrm{~mm}$ and $1 \mathrm{~mm}$ bands. As backends we used the wide bandwidth autocorrelator WILMA, which provides a spectral resolution of $2 \mathrm{MHz}$ and covers the whole band, and the narrow bandwidth correlator VESPA centered at the line frequency and providing a spectral resolution of $80 \mathrm{kHz}$ at $1.3 \mathrm{~mm}$ and $40 \mathrm{kHz}$ at $2.7 \mathrm{~mm}$ $\left(\sim 0.1 \mathrm{~km} \mathrm{~s}^{-1}\right)$. All observations were done using the wobbler switching (WS) procedure with a throw of $120^{\prime \prime}$. This procedure provides flat baselines, which are essential for detecting weak and wide lines toward compact sources, which is the case for the lines arising in circumstellar disks. In the case of AB Aur, the disk is still immersed in the parent cloud whose emission extends farther than the wobbler throw (see Semenov et al. 2005). Then, at the velocities of the ambient cloud the detected emission is just the ON-OFF balance without any physical interpretation (remember that the OFF position is moving in the sky during the source tracking). For this reason, we blanked the channels corresponding to the ambient cloud emission in the spectra toward AB Aur. We searched for $\mathrm{c}-\mathrm{C}_{3} \mathrm{H}_{2}$ and $\mathrm{SO}$ also toward DM Tau and $\mathrm{LkCa} 15$. In these cases, contamination from the ambient cloud is not expected. The observational results are shown in Table 2.

\section{Results}

In Fig. 1 we show some of the spectra observed toward AB Aur. The lines from the molecular cloud are very narrow, $\Delta v \sim$ $0.5 \mathrm{~km} \mathrm{~s}^{-1}$, and are centered at $5.9 \mathrm{~km} \mathrm{~s}^{-1}$ (Duvert et al. 1986 and Fig. 2a). The emission of the ambient cloud lies at the velocities $[5.4,6.4] \mathrm{km} \mathrm{s}^{-1}$. The channels corresponding to these velocities are blanked in the spectra shown in Fig. 1.
Table 2. Observational results.

\begin{tabular}{|c|c|c|c|c|c|c|}
\hline & Detectic & & & & on-detection & \\
\hline & $\begin{array}{l}\text { Line } \\
\qquad(\mathrm{mK} \times 1\end{array}$ & $\begin{array}{l}\text { Area }^{1} \\
\left.\mathrm{~m} \mathrm{~s}^{-1}\right)\end{array}$ & $\begin{array}{l}\mathrm{rms}^{2} \\
(\mathrm{mK})\end{array}$ & & Line & $\begin{array}{l}\mathrm{rms}^{2} \\
(\mathrm{mK})\end{array}$ \\
\hline & $\overline{\mathrm{AB} \mathrm{Al}}$ & & & & AB Aur & \\
\hline $\mathrm{HCO}^{+}$ & $1 \rightarrow 0$ & 47 & 4 & $\mathrm{CN}$ & $2 \rightarrow 1$ & 16 \\
\hline $\mathrm{HCO}^{+}$ & $3 \rightarrow 2$ & $932^{3}$ & $4^{3}$ & $\mathrm{CS}$ & $2 \rightarrow 1$ & 4 \\
\hline $\mathrm{CN}$ & $1 \rightarrow 0$ & 26 & 6 & $\mathrm{C}_{2} \mathrm{H}$ & $3 \rightarrow 2$ & 7 \\
\hline $\mathrm{CS}$ & $3 \rightarrow 2$ & 75 & 7 & $\mathrm{HCN}$ & $1 \rightarrow 0$ & 6 \\
\hline $\mathrm{H}_{2} \mathrm{CO}$ & $3_{0,3} \rightarrow 2_{0,2}$ & 87 & 5 & $\mathrm{c}-\mathrm{C}_{3} \mathrm{H}_{2}$ & $2 \rightarrow 1$ & 4 \\
\hline SO & $3_{4} \rightarrow 2_{3}$ & 26 & 4 & $\mathrm{c}-\mathrm{C}_{3} \mathrm{H}_{2}$ & $6 \rightarrow 5$ & 4 \\
\hline SO & $5_{6} \rightarrow 4_{5}$ & $64^{3}$ & $2^{3}$ & $\mathrm{DCN}$ & $2 \rightarrow 1$ & 4 \\
\hline $\mathrm{HCN}$ & $3 \rightarrow 2$ & 92 & 6 & $\mathrm{SiO}$ & $2 \rightarrow 1$ & 4 \\
\hline $\mathrm{DCO}^{+}$ & $2 \rightarrow 1$ & 8 & 2 & $\mathrm{SiO}$ & $6 \rightarrow 5$ & 9 \\
\hline $\mathrm{H}_{2} \mathrm{~S}$ & $1_{1,0} \rightarrow 1_{0,1}$ & 69 & 11 & $\mathrm{HCO}$ & $\begin{array}{l}1_{0,1} \rightarrow 0_{0.0} \\
\text { DM Tau }\end{array}$ & 4 \\
\hline & & & & $\mathrm{c}-\mathrm{C}_{3} \mathrm{H}_{2}$ & $2 \rightarrow 1$ & 3 \\
\hline & & & & $\mathrm{c}-\mathrm{C}_{3} \mathrm{H}_{2}$ & $6 \rightarrow 5$ & 6 \\
\hline & & & & SO & $\begin{array}{c}5_{6} \rightarrow 4_{5} \\
\mathrm{LkCa} 15\end{array}$ & $3^{3}$ \\
\hline & & & & c- $\mathrm{C}_{3} \mathrm{H}_{2}$ & $2 \rightarrow 1$ & 3 \\
\hline & & & & $\mathrm{c}-\mathrm{C}_{3} \mathrm{H}_{2}$ & $6 \rightarrow 5$ & 9 \\
\hline & & & & SO & $5_{6} \rightarrow 4_{5}$ & $3^{3}$ \\
\hline
\end{tabular}

Notes. ${ }^{(1)}$ Sum of the integrated intensity area in the velocity intervals $[4.2,5.6]+[6.4,7.25] \mathrm{km} \mathrm{s}^{-1}$. ${ }^{(2)} \mathrm{rms}$ in a channel of $1 \mathrm{~km} \mathrm{~s}^{-1}$. (3) Observed only with a velocity resolution of $2.7 \mathrm{~km} \mathrm{~s}^{-1}$.
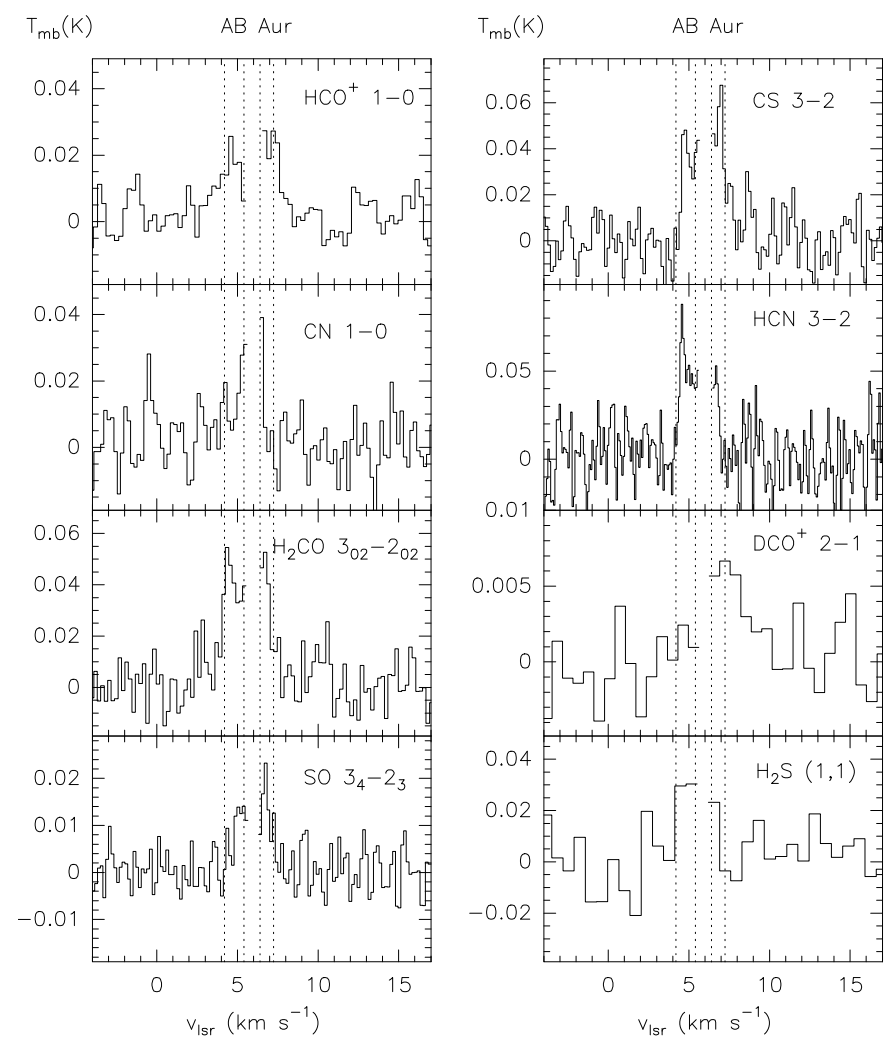

Fig. 1. Spectra obtained with the $30 \mathrm{~m}$ telescope toward AB Aur. Dashed lines indicate the velocities at which the disk emission arises $\left([4.2,5.6] \mathrm{km} \mathrm{s}^{-1}\right.$ and $\left.[6.5,7.25] \mathrm{km} \mathrm{s}^{-1}\right)$.

After blanking the cloud velocities, we detected emission at $>3 \sigma$ of the $\mathrm{HCO}^{+} 1 \rightarrow 0, \mathrm{CN} 1 \rightarrow 0, \mathrm{H}_{2} \mathrm{CO}_{3,3} \rightarrow 2_{0,2}, \mathrm{SO}_{4} \rightarrow$ $2_{3}$, CS $3 \rightarrow 2$, and $\mathrm{HCN} 3 \rightarrow 2$ lines. In addition, we tentatively detected $(\sim 3 \sigma)$ the $\mathrm{DCO}^{+} 2 \rightarrow 1$ and $\mathrm{H}_{2} \mathrm{~S} 1_{1,0} \rightarrow 1_{0,1}$ lines. All 

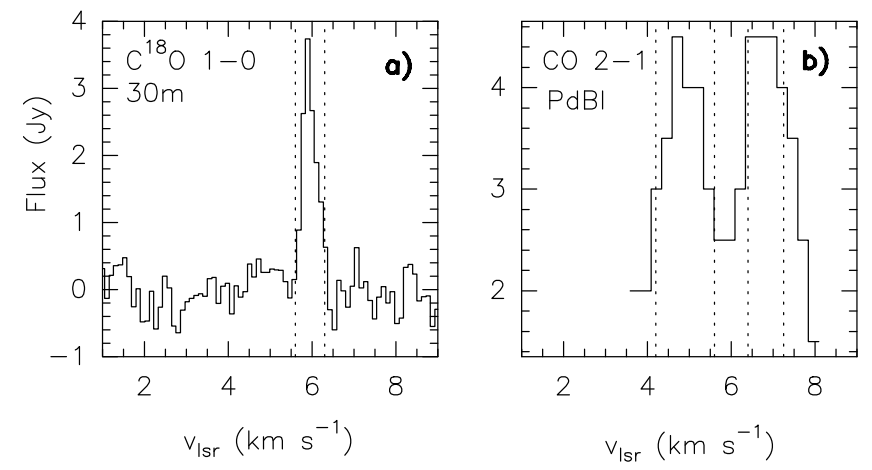

Fig. 2. a) Spectra of the $\mathrm{C}^{18} \mathrm{O} 1 \rightarrow 0$ line toward $\mathrm{AB}$ Aur observed with the IRAM $30 \mathrm{~m}$ telescope by Fuente et al. (2002). We adopted this profile as a pattern profile for the ambient cloud emission. Vertical lines indicate the velocity interval $[5.6,6.4] \mathrm{km} \mathrm{s}^{-1}$. b) Interferometric spectra of the ${ }^{12} \mathrm{CO} 2 \rightarrow 1$ toward the star position (Fig. 2 (bottom) of Piétu et al. 2005). Note that the disk emission occurs at the velocity intervals, $[4.2,5.6] \mathrm{km} \mathrm{s}^{-1}$ and $[6.4,7.25] \mathrm{km} \mathrm{s}^{-1}$. These velocities are indicated by vertical dashed lines.

the $(>3 \sigma)$ detected lines have the typical two-horn profile observed in the lines coming from the circumstellar disk, with two peaks centered at $4.8 \pm 0.25 \mathrm{~km} \mathrm{~s}^{-1}$ and $6.8 \pm 0.25 \mathrm{~km} \mathrm{~s}^{-1}$ (see Figs. 1 and 2b). This prompts us to interpret the emission of these lines as arising from the circumstellar disk. The only doubtful case is the $\mathrm{CN} 1 \rightarrow 0$ line, in which the two-horn profile is not as clear. Because $\mathrm{CN}$ is one of the most abundant species in disks (Dutrey et al. 1997; Thi et al. 2004; Öberg et al. 2010), we decided to keep it in our list of detected species. The narrowness of the $\mathrm{CN} 1 \rightarrow 0$ line could be because its emission is coming from the outermost part of the disk. We recall, however, that this detection requires further confirmation by interferometric observations.

The disk was previously detected in the $\mathrm{HCO}^{+} 1 \rightarrow 0$ line using the PdBI by Schreyer et al. (2008). Therefore, we can use this line to check the validity of our interpretation. In Fig. 3 we compare our $\mathrm{HCO}^{+}$spectrum with that observed toward the star position by Schreyer et al. (2008). Because the synthesized beam of these observations was $5.2^{\prime \prime} \times 4.8^{\prime \prime}$, this spectrum missed the emission of the outer part of the disk $(R>$ $378 \mathrm{AU})$. The emission of this outer region is expected at velocities $<0.87 \mathrm{~km} \mathrm{~s}^{-1}$ from the systemic velocity. We only consider velocities $>0.45 \mathrm{~km} \mathrm{~s}^{-1}$ relative to the systemic velocity, therefore the outer part of the disk is not relevant in our comparison. The integrated intensity emission of the $30 \mathrm{~m}$ spectra in the velocity intervals $[4.2,5.4] \mathrm{km} \mathrm{s}^{-1}$ and $[6.4,7.25] \mathrm{km} \mathrm{s}^{-1}$ is lower by a factor of $\approx 1.3$ than the integrated emission of the $\mathrm{HCO}^{+} 1$ $\rightarrow 0$ line as observed with the PdBI (see Fig. 3). The agreement is acceptable and we consider that our interpretation is valid.

In Table 2, we show the list of non-detections (in the considered velocity ranges). Our $3 \sigma$ upper limit to the emission of CS $2 \rightarrow 1$ line improves by a factor of 2 the previous one obtained by Schreyer et al. (2008) using the PdBI.

\section{Averaged molecular abundances in the disk}

Below we derive approximated average column densities in the disks assuming optically thin emission, a uniform temperature of $T_{\mathrm{k}}=10 \mathrm{~K}$ and $20 \mathrm{~K}$, and local thermodynamic equilibrium (LTE). The assumed disk sizes (diameters) are 13" for AB Aur (Piétu et al. 2005), 6" for LkCa15 (Thi et al. 2004) and 13" for DM Tau (Piétu et al. 2007). In Table 3 we compare the

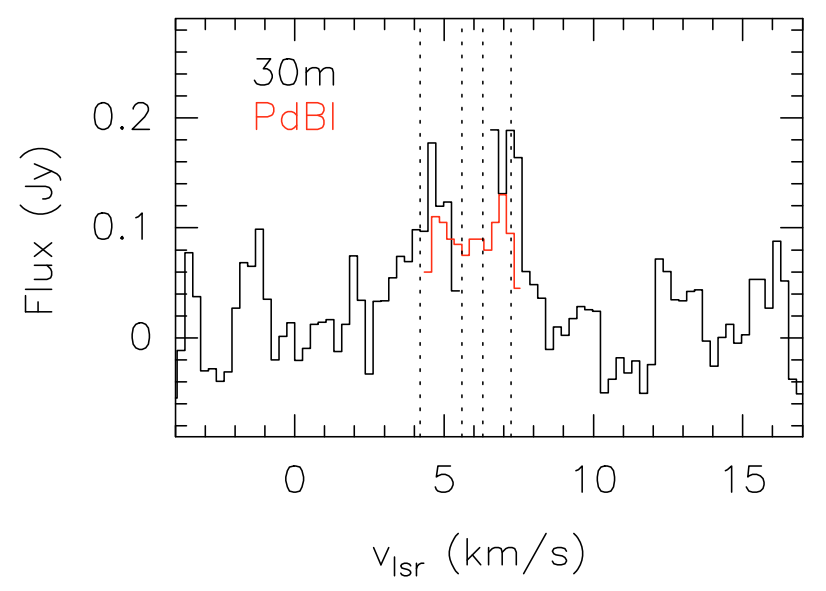

Fig. 3. Comparison between the spectra of the $\mathrm{HCO}+1 \rightarrow 0$ line observed toward AB Aur with the $30 \mathrm{~m}$ telescope and the PdBI. Dashed lines indicate the velocities at which the disk emission arises ([4.2, $5.6] \mathrm{km} \mathrm{s}^{-1}$ and $[6.5,7.25] \mathrm{km} \mathrm{s}^{-1}$ ).

obtained fractional abundances with those derived in other disks following a similar procedure. The first result is that the molecular abundances measured toward $\mathrm{AB}$ Aur are very similar to those found toward other disks, which reinforces our interpretation of the disk origin for the observed lines.

The $\mathrm{HCO}^{+}$abundance in AB Aur is similar to those measured in the TT stars LkCa15 and TW Hya, and in the HAe stars HD 163296 and MWC 480. Only DM Tau presents a significantly higher (a factor of 10) $\mathrm{HCO}^{+}$abundance, which suggests that DM Tau is a special case among circumstellar disks. The same is true for $\mathrm{CN}$ and $\mathrm{HCN}$. Both molecules present abundances of $\sim 10^{-10}(\mathrm{CN})$ and $\sim 10^{-11}$ (HCN) in all the disks except DM Tau, in which the measured abundances are a factor of 10 higher. The case of $\mathrm{H}_{2} \mathrm{CO}$ is a bit different. It is also overabundant in DM Tau $\left(5 \times 10^{-10}\right)$ but it is underabundant in TW Hya $\left(<10^{-12}\right)$. Our SO detection in AB Aur is the first one in a circumstellar disk and so is our estimate of the SO abundance in a circumstellar disk. We unsuccessfully searched for SO toward DM Tau and LkCa15. Our upper limit toward DM Tau shows that in contrast with the behavior observed in the other species, $\mathrm{SO}$ is not overabundant in DM Tau relative to AB Aur. The abundance of $\mathrm{C}_{2} \mathrm{H}$ in $\mathrm{AB}$ Aur is 1000 times lower than in DM Tau.

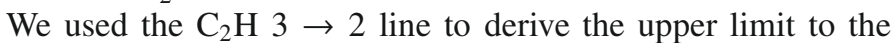
$\mathrm{C}_{2} \mathrm{H}$ abundance. Recent results by Henning et al. (2010) show that the excitation temperature of $\mathrm{C}_{2} \mathrm{H}$ could be lower than $10 \mathrm{~K}$ in some disks. Then, the value of the upper limit would increase. The searched $\mathrm{c}-\mathrm{C}_{3} \mathrm{H}_{2}$ line has not been detected in any of the observed disks. The obtained upper limit to the $\left[\mathrm{c}-\mathrm{C}_{3} \mathrm{H}_{2}\right] /\left[\mathrm{HCO}^{+}\right]$ ratio is still consistent with the observational results in PDRs (Fuente et al. 2003).

In Table 3 we also compare some representative column density ratios. These ratios are meaningful only provided that the molecules arise in the same region. The value of $[\mathrm{CN}] /[\mathrm{HCN}]$ is fairly uniform (within a factor of 2) among the observed disks. However, there are large variations, more than one order of magnitude, in the values of $[\mathrm{CS}] /[\mathrm{SO}],\left[\mathrm{H}_{2} \mathrm{CO}\right] /\left[\mathrm{HCO}^{+}\right]$ and $\left[\mathrm{C}_{2} \mathrm{H}\right] /\left[\mathrm{HCO}^{+}\right]$. This suggests that these column density ratios are more sensitive to the in most cases poorly known disk structure and/or grain properties. One important parameter in disks is the deuteration degree. Qi et al. (2008) derived a $\left[\mathrm{DCO}^{+}\right] /\left[\mathrm{HCO}^{+}\right]$ratio of $\sim 0.03$ in TW Hya. Our tentative detection of $\mathrm{DCO}^{+}$in $\mathrm{AB}$ Aur would imply a $\left[\mathrm{DCO}^{+}\right] /\left[\mathrm{HCO}^{+}\right]$ratio of $\sim 0.03$, similar to TW Hya. 
Table 3. Comparison with other disks ${ }^{(1)}$.

\begin{tabular}{|c|c|c|c|c|c|c|c|}
\hline & \multicolumn{2}{|c|}{ AB Aur } & \multirow[t]{2}{*}{ DM Tau } & \multirow[t]{2}{*}{$\mathrm{LkCa} 15$} & \multirow[t]{2}{*}{ TW Hya } & \multirow{2}{*}{ HD163296 } & \multirow{2}{*}{ MWC 480} \\
\hline & $T_{\mathrm{rot}}=10 \mathrm{~K}$ & $T_{\text {rot }}=20 \mathrm{~K}$ & & & & & \\
\hline $\mathrm{X}\left(\mathrm{HCO}^{+}\right)^{(1)}$ & $1.8 \times 10^{-11}$ & $2.8 \times 10^{-11}$ & $7.4 \times 10^{-10}$ & $5.6 \times 10^{-12}$ & $2.2 \times 10^{-11}$ & $7.8 \times 10^{-12}$ & $1.0 \times 10^{-10}$ \\
\hline $\mathrm{X}(\mathrm{CN})$ & $1.0 \times 10^{-10}$ & $1.4 \times 10^{-10}$ & $3.2 \times 10^{-9}$ & $2.4 \times 10^{-10}$ & $1.2 \times 10^{-10}$ & $1.3 \times 10^{-10}$ & $1.4 \times 10^{-10}$ \\
\hline $\mathrm{X}(\mathrm{CS})$ & $3.6 \times 10^{-11}$ & $3.4 \times 10^{-11}$ & $3.3 \times 10^{-10}$ & $<8.5 \times 10^{-11}$ & & & \\
\hline $\mathrm{X}\left(\mathrm{C}_{2} \mathrm{H}\right)$ & $<2.1 \times 10^{-11}$ & $<1.1 \times 10^{-11}$ & $1.1 \times 10^{-8}$ & & & & \\
\hline $\mathrm{X}\left(\mathrm{H}_{2} \mathrm{CO}\right)$ & $4.5 \times 10^{-11}$ & $5.3 \times 10^{-11}$ & $5.0 \times 10^{-10}$ & & $<1.4 \times 10^{-12}$ & $1.0 \times 10^{-11}$ & $<1.4 \times 10^{-11}$ \\
\hline $\mathrm{X}(\mathrm{SO})$ & $4.4 \times 10^{-11}$ & $4.6 \times 10^{-11}$ & $<3.0 \times 10^{-11}$ & $<2.0 \times 10^{-11}$ & $<4.1 \times 10^{-11}$ & & \\
\hline $\mathrm{X}(\mathrm{HCN})$ & $1.7 \times 10^{-11}$ & $1.0 \times 10^{-11}$ & $4.9 \times 10^{-10}$ & $3.1 \times 10^{-11}$ & $1.6 \times 10^{-11}$ & $<9.1 \times 10^{-12}$ & $<1.1 \times 10^{-11}$ \\
\hline $\mathrm{X}\left(\mathrm{c}-\mathrm{C}_{3} \mathrm{H}_{2}\right)$ & $<2.4 \times 10^{-11}$ & $<1.0 \times 10^{-11}$ & $<1.3 \times 10^{-11}$ & $<1.3 \times 10^{-11}$ & & & \\
\hline $\mathrm{X}\left(\mathrm{DCO}^{+}\right)$ & $7.1 \times 10^{-13}$ & $7.9 \times 10^{-13}$ & & $<7.9 \times 10^{-13}$ & $7.8 \times 10^{-13}$ & & \\
\hline $\mathrm{X}(\mathrm{DCN})$ & $<3.8 \times 10^{-12}$ & $<4.3 \times 10^{-12}$ & & & & & \\
\hline $\mathrm{X}(\mathrm{SiO})$ & $<1.7 \times 10^{-11}$ & $<2.4 \times 10^{-11}$ & & & & & \\
\hline $\mathrm{X}(\mathrm{HCO})$ & $<1.9 \times 10^{-10}$ & $<3.9 \times 10^{-10}$ & & & & & \\
\hline $\mathrm{X}\left(\mathrm{H}_{2} \mathrm{~S}\right)$ & $8.3 \times 10^{-11}$ & $6.3 \times 10^{-11}$ & & & & & \\
\hline $\mathrm{X}(\mathrm{CN}) / \mathrm{X}(\mathrm{HCN})$ & 6 & 14 & 6 & 8 & 7 & $>14$ & $>13$ \\
\hline $\mathrm{X}(\mathrm{CS}) / \mathrm{X}(\mathrm{SO})$ & 0.8 & 0.7 & $>11$ & & & & \\
\hline $\mathrm{X}\left(\mathrm{DCO}^{+}\right) / \mathrm{X}\left(\mathrm{HCO}^{+}\right)$ & 0.04 & 0.03 & & & 0.03 & 0.004 & \\
\hline $\mathrm{X}\left(\mathrm{H}_{2} \mathrm{CO}\right) / \mathrm{X}\left(\mathrm{HCO}^{+}\right)$ & 2.5 & 2 & 0.7 & & $<0.06$ & 1.3 & $<0.14$ \\
\hline $\mathrm{X}\left(\mathrm{C}_{2} \mathrm{H}\right) / \mathrm{X}\left(\mathrm{HCO}^{+}\right)$ & $<1$ & $<0.4$ & 15 & & & & \\
\hline
\end{tabular}

Notes. ${ }^{(1)}$ Abundances relative to $\mathrm{H}_{2}$ were calculated assuming a disk diameter of 13" for AB Aur (Piétu et al. 2005), 6" for LkCa15 (Thi et al. 2004) and 13" for DM Tau (Piétu et al. 2007). The assumed disk-averaged molecular hydrogen column densities are $\mathrm{N}\left(\mathrm{H}_{2}\right)=1.1 \times 10^{22} \mathrm{~cm}^{-2}$ for AB Aur, $2.7 \times 10^{22} \mathrm{~cm}^{-2}$ for DM Tau and $1.4 \times 10^{23} \mathrm{~cm}^{-2}$ for LkCa15. In our calculations for DM Tau and LkCa15, we adopt $T_{\text {rot }}=10 \mathrm{~K}$. Abundances for the other disks/molecules are taken from Dutrey et al. (1997) (for DM Tau), Thi et al. (2004) and Guilloteau et al. (2006).

\section{Discussion}

In order to guide our interpretation of the observed features and provide additional support to their disk origin, we performed a (preliminary) chemical model adopting the disk and stellar parameters from Schreyer et al. (2008) and the updated chemical network of Agúndez et al. (2008). Our aim is to investigate if detectable SO column densities can be produced in this disk. In Fig. 4 we show the radial distribution of the vertical column densities of some molecules as calculated at 2.5 Myr (the age of AB Aur). In agreement with the observational results of Piétu et al. (2005), the intense stellar radiation makes the disk moderately warm, with temperatures above $20 \mathrm{~K}$ even in the disk midplane, so that volatile molecules such as $\mathrm{CO}$ are not severely depleted on grain surfaces. In our model, the major gas phase reservoir of sulphur are the CS and SO molecules, with high column densities of SO mainly present in the inner $R<200 \mathrm{AU}$ region of the disk. The SO abundance decreases rapidly with time because of the adsorption onto the grain surfaces. The youth of the AB Aur disk could be key for higher SO abundance.

\section{Summary and conclusions}

We have taken advantage of the high sensitivity of the EMIR receivers recently installed in the IRAM 30 m telescope to make a sensitive search for molecular emission in three prototypical disks, AB Aur, DM Tau, and LkCa 15. Our results and conclusions can be summarized as follows:

- We detected the $\mathrm{HCO}^{+} 1 \rightarrow 0, \mathrm{CN} 1 \rightarrow 0, \mathrm{H}_{2} \mathrm{CO} 3_{0,3} \rightarrow$ $2_{0,2}, \mathrm{SO} 3_{4} \rightarrow 2_{3}, \mathrm{CS} 3 \rightarrow 2, \mathrm{HCN} 1 \rightarrow 0$ and $\mathrm{HCN} 3 \rightarrow$ 2 lines toward $\mathrm{AB}$ Aur. In addition, we tentatively detected the $\mathrm{DCO}^{+} 2 \rightarrow 1$ and $\mathrm{H}_{2} \mathrm{~S} 1_{1,0} \rightarrow 1_{0,1}$ lines. Based on the lines profiles, we interpret the emission of the $\mathrm{CN} 1 \rightarrow 0$, $\mathrm{HCN} 3 \rightarrow 2, \mathrm{H}_{2} \mathrm{CO}_{30,3} \rightarrow 2_{0,2}, \mathrm{CS} 3 \rightarrow 2$, and the SO $3_{4} \rightarrow$ 2 lines as arising from the disk. If confirmed, this is the first detection of SO in a circumstellar disk.

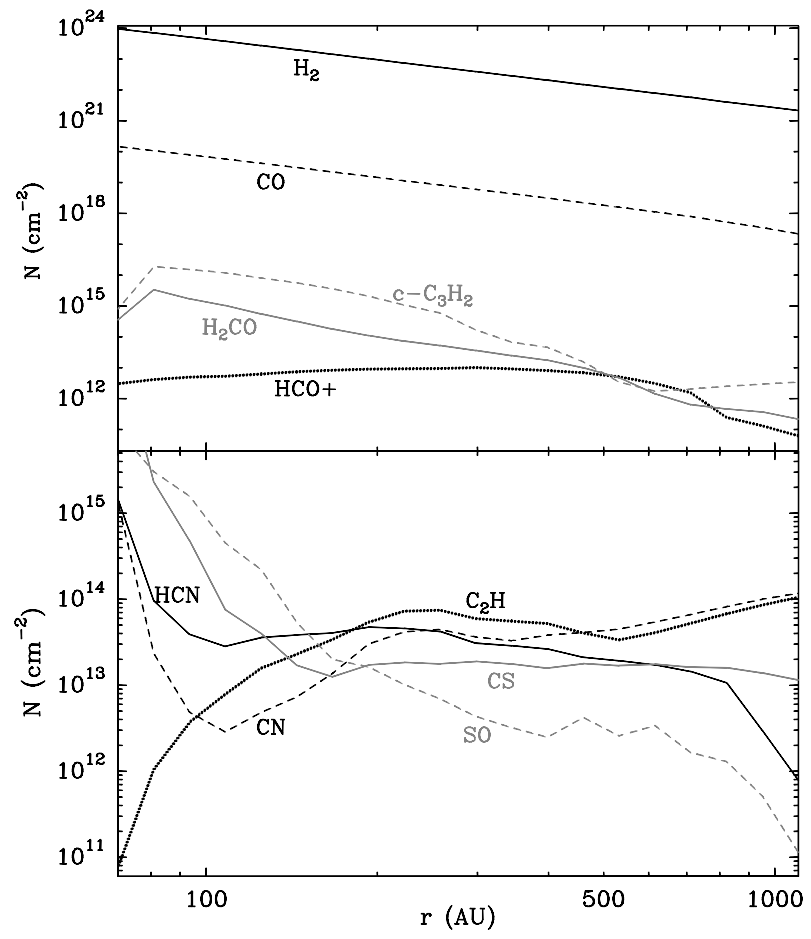

Fig. 4. Vertical column densities of various molecules as a function of radius at $2.5 \mathrm{Myr}$ as calculated by the chemical model.

- We have unsuccessfully searched for SO toward DM Tau and LkCa 15. The obtained upper limits show that SO is underabundant in DM Tau compared to AB Aur.

- We have searched for $\mathrm{c}-\mathrm{C}_{3} \mathrm{H}_{2}$ toward AB Aur, DM Tau and LkCa 15. The obtained upper limits are still consistent with the $\left[\mathrm{c}-\mathrm{C}_{3} \mathrm{H}_{2}\right] /\left[\mathrm{HCO}^{+}\right]$values obtained in PDRs.

Our observational work has significantly increased (from 1 to 6 ) the number of species detected toward the disk in AB Aur. 
If confirmed by interferometric observations, the SO detection would be the first one in a circumstellar disk. Our chemical model suggests that the high SO abundance derived in AB Aur disk is consistent with that expected in a very young and warm disk, where depletion of gas onto grains is not yet dominating the chemistry.

Acknowledgements. This paper has been partially supported by MICINN under grant AYA2009-07304 and within the program CONSOLIDER INGENIO 2010, under grant "Molecular Astrophysics: The Herschel and ALMA Era ASTROMOL" (ref.: CSD2009-00038). M.A. is supported by a Marie Curie Intra-European Individual Fellowship within the European Community 7th Framework Programme under grant agreement no. 235753. J.R.G. was supported by a Ramón y Cajal research contract from the Spanish MICINN and co-financed by the European Social Fund.

\section{References}

Aikawa, Y., \& Herbst, E. 2000, From Molecular Clouds to Planetary, 197, 425 Agúndez, M., Cernicharo, J., \& Goicoechea, J. R. 2008, A\&A, 483, 831
Dutrey, A., Guilloteau, S., \& Guelin, M. 1997, A\&A, 317, L55

Dutrey, A., Henning, T., Guilloteau, S., et al. 2007, A\&A, 464, 615

Duvert, G., Cernicharo, J., \& Baudry, A. 1986, A\&A, 164, 349

Fuente, A., Martín-Pintado, J., Bachiller, R., Rodríguez-Franco, A., \& Palla, F. 2002, A\&A, 387, 977

Fuente, A., Rodríguez-Franco, A., García-Burillo, S., Martín-Pintado, J., \& Black, J. H. 2003, A\&A, 406, 899

Guilloteau, S., Piétu, V., Dutrey, A., \& Guélin, M. 2006, A\&A, 448, L5

Henning, Th., Semenov, D., Guilloteau, St., et al. 2010, ApJ, 714, 1511

Hernández, J., Calvet, N., Briceño, C., Hartmann, L., \& Berlind, P. 2004, AJ, 127,1682

Nomura, H., Aikawa, Y., Nakagawa, Y., \& Millar, T. J. 2009, A\&A, 495, 183

Öberg, K. I., Qi, C., Fogel, J. K. J., et al. 2010, ApJ, accepted [arXiv: 1007. 1476]

Piétu, V., Guilloteau, S., \& Dutrey, A. 2005, A\&A, 443, 945

Piétu, V., Dutrey, A., \& Guilloteau, S. 2007, A\&A, 467, 163

Qi, C., Wilner, D. J., Aikawa, Y., Blake, G. A., \& Hogerheijde, M. R. 2008, ApJ, 681,1396

Schreyer, K., Guilloteau, S., Semenov, D., et al. 2008, A\&A, 491, 821

Semenov, D., Pavlyuchenkov, Y., Schreyer, K., et al. 2005, ApJ, 621, 853

Thi, W.-F., van Zadelhoff, G.-J., \& van Dishoeck, E. F. 2004, A\&A, 425, 955

van den Ancker, M. E., de Winter, D., \& Tjin A Djie, H. R. E. 1998, A\&A, 330, 145 